\title{
Two patients with glutaric aciduria type 3: a novel mutation and brain magnetic resonance imaging findings
}

\author{
Sevil Dorum ${ }^{1 \oplus}$, Cengiz Haval1 ${ }^{2 \oplus}$, Özlem Görükmez ${ }^{3 \oplus}$, Orhan Görükmez ${ }^{3 \oplus}$ \\ Divisions of ${ }^{1}$ Metabolism Unit and ${ }^{2}$ Pediatric Neurology, Department of Pediatrics, ${ }^{3}$ Department of Genetics, Bursa Yuksek Ihtisas \\ Training and Research Hospital, Bursa, Turkey.
}

\begin{abstract}
Background. Glutaric Aciduria Type 3 (GA-3) is a rare metabolic disease which is inherited autosomal recessively and characterized by isolated glutaric acid excretion. To date, a limited number of cases have been reported in the literature. We present two patients with GA3 who were diagnosed with the isolated increased level of glutaric acid in urine.

Case. Glutaric aciduria type 1 and type 2 were excluded by genetic analysis and other laboratory and clinical findings. One of our patients had a homozygous mutation p.Arg322Trp (c.964C> T) of SUGCT (NM_001193311) gene. To the best of our knowledge this mutation has not been reported in the literature previously. Symmetrical periventricular and deep cerebral white matter abnormalities were detected on his brain magnetic resonance imaging (MRI).
\end{abstract}

Conclusion. We present two patients with GA-3 and a novel mutation in the SUGCT gene. Our findings expand the spectrum of causative mutations and clinical findings in GA-3.

Key words: brain magnetic resonance imaging, glutaric aciduria type 3, novel mutation, SUGCT gene.

Glutaric aciduria type 3(GA-3) is a rare metabolic disorder caused by variants of the SUGCT (C7orf10) gene. It is characterized by the reduced transformation of free glutaric acid to glutarylcoA due to succinate-hydroxymethylglutarateCoA-transferase enzyme deficiency. This deficiency leads to isolated glutaric acid accumulation or excretion. ${ }^{1,2}$

While other types of glutaric acidemia have been known as mitochondrial disease, it has been accepted that GA-3 is caused by a peroxisomal dysfunction. In contrast to, glutaric aciduria type types 1 (GA-1) and 2 (GA-2), GA-3 is less known and rarely occurs. Some authors have suggested that GA-3 is not a disease. ${ }^{3}$ There is limited knowledge regarding the disease that is composed of a several case reports in

Sevil Dorum

sevildorum@gmail.com

Received 18th February 2020, revised 29th March 2020, 4th April 2020, accepted 6th April 2020. the literature. Additionally, nearly half of patients with GA3 that have been reported in the literature were asymptomatic and were identified through the newborn screening programme..$^{1-6}$

In this report, two patients with GA3 who had distinct genotypes and phenotypes have been presented. Furthermore, one of the patients had brain magnetic resonance imaging (MRI) findings we believe were previously undefined.

\section{Case 1}

An 11-year-old female patient was admitted for sensorineural hearing loss and intellectual disability. She had a seizure at the age of 1 year. Her parents are from a consanguineous marriage (Fig. 1A). She had been diagnosed with global developmental delay and hearing loss at the age of 2 years. She had intellectual and speech impairments. Systemic physical examination was otherwise normal. Plasma 
acylcarnitine, and amino acid profiles were unremarkable. Increased glutaric acid levels were noticed in urine organic acid profiles (108 mg/g creatinine). In the genetic analysis of SUGCT (NM_001193311) gene, Arg108 (c.322C> T) mutation was homozygous (Fig. 1B). Riboflavin (vitamin B2) treatment was started at $200 \mathrm{mg} /$ day. No laboratory and clinical changes were observed with riboflavin (vitamin B2) treatment.

Clinical exome sequencing and Sanger sequencing were applied for both of the patients. Genomic DNAs were extracted from peripheral venous blood using the QIAamp ${ }^{\circledR}$ DNA Mini Kit (QIAGEN, Ankara, Turkey). The Clinical Exome Solution (SOPHiA GENETICS, Switzerland) was used for exome enrichment. All procedures were carried out according to the manufacturer's protocols. It is a capturebased target enrichment kit and covers 4,900 genes with known inherited disease causing mutations.

Next generation sequencing showed homozygous nonsense mutation, c.322 $>\mathrm{T}$, p.Arg108* in the SUGCT (NM_001193311) gene, and homozygous missense mutation, c.250C $>\mathrm{T}$,
p.Arg84Trp in TMIE (NM_147196) gene and these mutations were confirmed by sanger sequencing in this family (Fig. 1B).

\section{Case 2}

A 13-month old male, was admitted due to neuromotor developmental delay and tremor. He was born at 40 weeks gestational ages with a birth weight of 2200 grams. He was the third child of healthy consanguineous Syrian parents. He had two healthy siblings. His family history and antenatal, perinatal and postnatal histories were unremarkable. He had seizures in clusters at four months of age for three days in the course of acute gastroenteritis. Brain MRI and electroencephalography were normal during that period. The patient sat with support at the age of 7 months, and he did not achieve the ability to sit without support at the age of 1 year. At the age of 13 months, subacute regression in his motor abilities occurred. On examination, his weight, height and head circumference were $<3$ rd percentile. He had axial hypotonia and bilaterally clonus and spasticity. The patients' ability to sit with support regressed. Blood gas, blood ketone level, dry blood acylcarnitine and
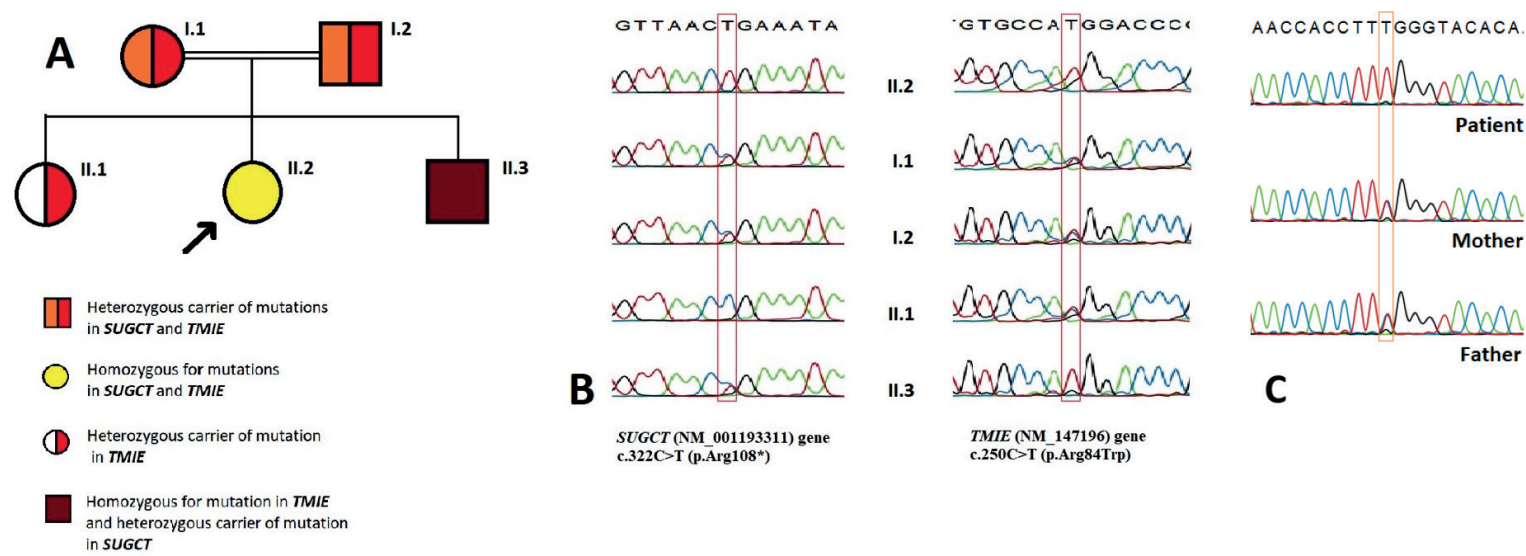

Fig. 1. Pedigree of the family showing the identified variants and mutation analysis

[A]. Shown is a consanguineous family segregating Autosomal Recessive Deafness 6 (DFNB6) and Glutaric Aciduria III (GA III). The arrow indicates the proband.

[B]. Electropherograms of Sanger's sequencing analysis of the family. Germline mutations in SUGCT and TMIE genes (indicated by red frames).

[C]. Result of DNA sequencing of the second family. A novel homozygous germline mutation, c.964C>T, p.Arg322Trp in SUGCT gene of the case 2 (indicated by red frame). 
blood amino acid profile were normal. Auditory Brainstem Response test was normal. There was an increase in the level of glutaric acid in urine organic acids to $26.4 \mathrm{mg} / \mathrm{g}$ creatinine $(\mathrm{N}<5 \mathrm{mg} / \mathrm{g}$ creatinine). Molecular analysis of the SUGCT (NM_001193311) gene showed the homozygous variant of p.Arg322Trp (c.964C> $\mathrm{T}$ ), not previously reported in the literature (Fig. $1 C)$.

In this case, we detected a homozygous missense mutation, c.964C $>$ T, p.Arg322Trp in the SUGCT (NM_001193311) gene, which was confirmed by sanger sequencing as mentioned before. This variant has not been previously reported in the Human Gene Mutation Database (HGMD; http://www.hgmd.cf.ac.uk/ac/index.php) and in 1000 Genomes Project. Silico analysis programs (MutationTaster; disease causing, PolyPhen2; probably damaging, and SIFT; damaging) showed that this change could be the cause of the disease. We also showed the same mutation as heterozygous in the parents (Fig. 1C).

On the brain MRI, there were extensive, symmetrical periventricular and deep cerebral white matter abnormalities with sparing of the $U$ fibers of the cerebral hemispheres. The internal capsule, corticospinal tracts in the brain stem and the cerebellar white matter were normal (Fig. 2). Carnitine $(50 \mathrm{mg} / \mathrm{kg} /$ day), and riboflavin (100 mg/day) treatments were started. Lorazepam and physical therapy were initiated for spasticity. During follow-up, the patients' weight increased. His spasticity resolved. Motor development and mobility of patient moderately ameliorated.

Informed consent was received from both of family.

\section{Discussion}

In this article, two patients with GA-3 who were diagnosed with urine organic acids and genetic tests were presented. GA-3 remains less described and known in comparison with other types of glutaric aciduria. In the literature only a few cases have been reported to date and most cases were asymptomatic and were revealed by a screening-programme. The remaining symptomatic patients with GA-3 presented with different clinical and laboratory findings such as diarrhea, vomiting, cyclic vomiting attacks, metabolic acidosis, ketosis, and hypoglycemia. ${ }^{1,2,6}$

Our first case was evaluated with intellectual disability and sensorineural hearing loss. In this case, sensorineural hearing loss was thought to be associated with a concomitant additional mutation in the TMIE gene. Brain MRI could not be performed because of the cochlear implant. The patient was also started on riboflavin treatment.

Our second case had neuromotor delay and hypotonia, similar to the cases previously described in the literature. ${ }^{7}$ On brain MRI of a few patients previously reported in the literature, nonspecific white matter changes were described. ${ }^{1-3,6}$ On brain MRI of our patient, there were extensive, symmetrical periventricular and deep cerebral white matter abnormalities with sparing of the $U$ fibers of the cerebral hemispheres. These findings have not previously been reported to be associated with GA3 in the literature.

GA-1 and GA-2 that are present with increased glutaric acid in urine have relatively different clinical findings and distinctive patterns of involvement on brain MRI. GA-1 has a highly variable clinical manifestation. It frequently occurs with acute encephalitis-like encephalopathy in early childhood. It is often precipitated with gastroenteritis, febrile illness, and immunization. The typical neurological findings include dystonia, axial hypotonia, spasticity, akinetic-rigid parkinsonism. ${ }^{8}$ Enlarged cerebrospinal fluid spaces anterior and temporal areas, subdural collections, white matter involvement and cerebral atrophy, are included MRI findings in GA-1. ${ }^{9}$ Also, T2 hyperintensity of caudate and lentiform nuclei is frequently encountered on MRI. Corpus callosum, globus pallidus, dentate nuclei, and 

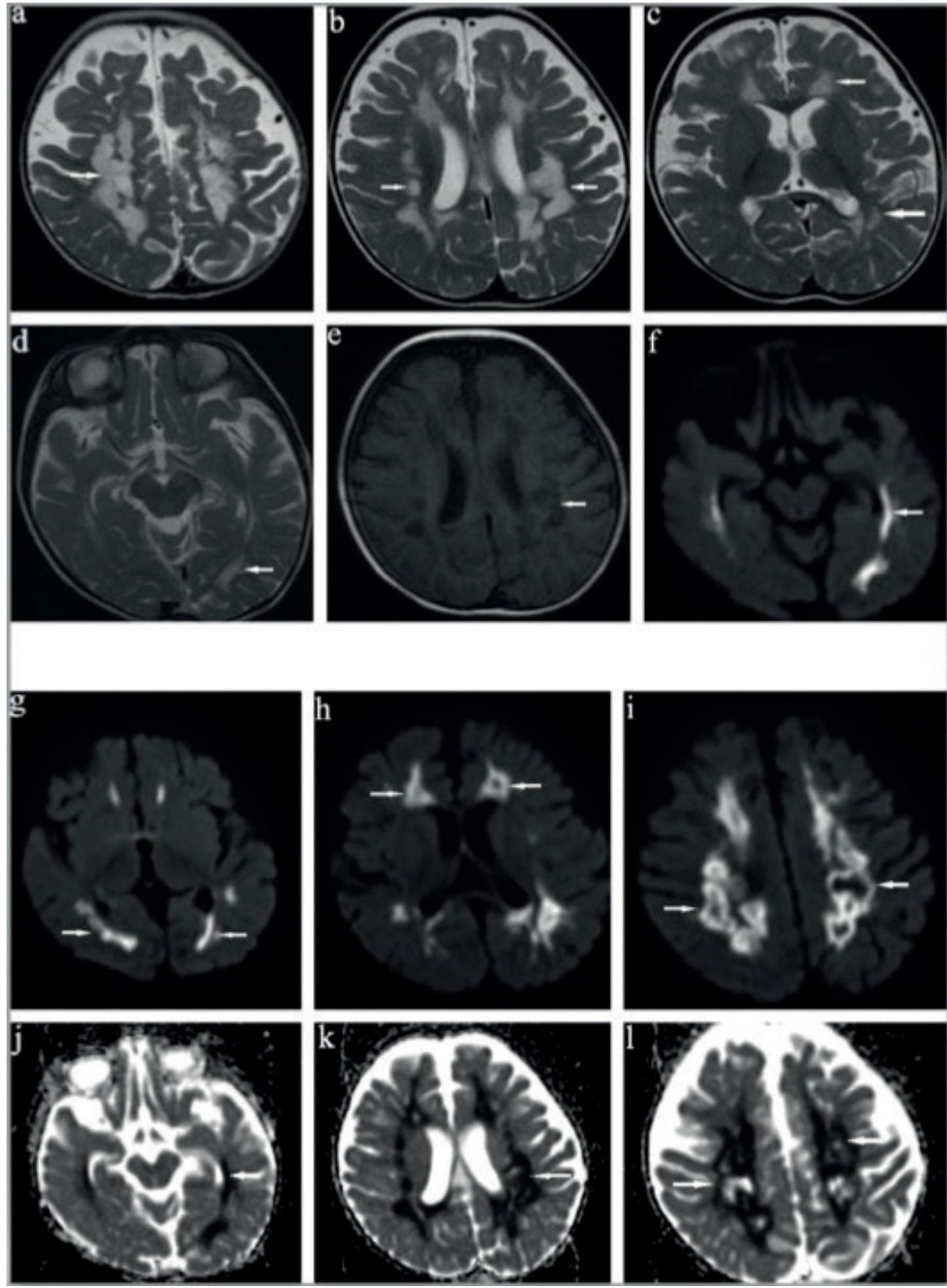

Fig. 2. There are extensive, symmetrical periventricular and deep cerebral white matter abnormalities with sparing of the $U$ fibers of the cerebral hemispheres. The internal capsule, corticospinal tracts in the brain stem and the cerebellar white matter are normal $(a, b, c, d)$. The T1-weighted images reveal that the involvement has an abnormally low signal intensity and cystic nature (e). Also, there are extensive signal abnormalities that refer to abnormal restricted diffusion in the diffusion-weighted imaging ( $f, g, h, i, j, k, l)$. 
substantia nigra may also participate in brain involvement in patients with GA- $1 .^{10}$

GA-2 appears with recurrent vomiting, lethargy, hypoglycemia, and metabolic acidosis. Lipid-storage myopathy is found of muscle involvement. Three distinct forms of GA-2 are described. Type I and Type II are neonatal-onset form and associated with nonketotic hypoglycemia, and metabolic acidosis. Type 1 also includes congenital anomalies. Type III is the late-onset form and moderately clinical form. ${ }^{11}$ Reported MRI findings include, leukodystrophy, recurrent episodes of CNS demyelination and bilaterally symmetrical T2 hyperintensities in the globus pallidus. ${ }^{12,13}$

In addition to GA-1 and GA-2, a group of mitochondrial disease should be considered in the differential diagnosis with patients who have symmetrical periventricular and deep cerebral white matter abnormalities on the brain MRI. The pattern of involvement, dysmyelinated areas of white matter, basal ganglia and cortical structures participation are noteworthy in the differential diagnosis. Complex I and II deficiency may lead to isolated symmetrical periventricular cystic involvement. Determinations of plasma and CSF lactate and pyruvate levels, blood gas analysis, analysis of urinary organic acids are essential hallmark tests. ${ }^{14}$ There was no mutation in the NDUFS4, SDHA, SDHD and SDHAF1 genes in the clinical exome analysis.

As recommended in the literature, we started therapy with carnitine, riboflavin. ${ }^{1,2}$ We have observed weight gain and an improvement in motor abilities in the case 2 .

GA-1 and GA-2 were excluded by genetic analysis in our patients who had high levels of glutaric acid in urine. Genetic analysis revealed homozygous variants in SUGCT (NM_001193311) genes. The homozygous variant p.Arg322Trp (c.964C> T) detected in the second patient was previously unreported.

In conclusion we present two patients with GA3 and a novel mutation in the SUGCT gene.
Our findings expand the spectrum of causative mutations and clinical findings in GA III.

\section{REFERENCES}

1. Waters PJ, Kitzler TM, Feigenbaum A, et al. Glutaric aciduria type 3: three unrelated Canadian cases, with different routes of ascertainment. JIMD Rep 2018; 39: 89-96.

2. Bennett MJ, Pollitt RJ, Goodman SI, Hale DE, Vamecq J. Atypical riboflavin-responsive glutaric aciduria, and deficient peroxisomal glutaryl-CoA oxidase activity: a new peroxisomal disorder. J Inherit Metab Dis 1991; 14: 165-173

3. Knerr I, Zschocke J, Trautmann U, et al. Glutaric aciduria type III: a distinctive non-disease? J Inherit Metab Dis 2002; 25: 483-490.

4. Marlaire S, Van Schaftingen E, Veiga-da-Cunha M. C7orf10 encodes succinate-hydroxymethylglutarate CoA-transferase, the enzyme that converts glutarate to glutaryl-CoA. J Inherit Metab Dis 2014; 37: 13-19.

5. Sherman EA, Strauss KA, Tortorelli S, et al. Genetic mapping of glutaric aciduria, type 3, to chromosome 7 and identification of mutations in C7orf10. Am J Hum Genet 2008; 83: 604-609.

6. Skaricic A, Zekusic M, Fumic K, et al. New symptomatic patients with glutaric aciduria type 3 : further evidence of high prevalence of the c.1006C $>\mathrm{T}$ (p.Arg336Trp) mutation. J Inherit Metab Dis 2016; 39(Suppl 1): S1-S34.

7. Robinson JT, Thorvaldsdóttir H, Winckler W, et-al. Integrative genomics viewer. Nat Biotechnol 2011; 29: 24-26.

8. Nunes J, Loureiro S, Carvalho S, et al. Brain MRI findings as an important diagnostic clue in glutaric aciduria type 1. Neuroradiol J 2013; 26: 155-161.

9. Brismar J, Ozand PT. CT and MR of the brain in glutaric acidemia type 1: a review of 59 published cases and report of 5 new patients. AJNR Am J Neuroradiol 1995; 16: 675-683.

10. Twomey EL, Naughten ER, Donoghue VB, Ryan S. Neoroimaging findings in glutaric aciduria type 1 . Pediatr Radiol 2003; 33: 823-830.

11. Loehr JP, Goodman SI, Frerman FE. Glutaric acidemia type II: heterogeneity of clinical and biochemical phenotypes. Pediatr Res 1990; 27: 311315.

12. Ishii K, Komaki H, Ohkuma A, Nishino I, Nonaka I, Sasaki M. Central nervous system and muscle involvement in an adolescent patient with riboflavinresponsive multiple acyl-CoA dehydrogenase deficiency. Brain Dev 2010; 328: 669-672. 
13. Mumtaz HA, Gupta V, Singh P, Marwaha RK, Khandelwal N. MR imaging findings of glutaric aciduria type II. Singapore Med J 2010; 51: e69-e71.
14. van der Knaap MS, Valk J. Leigh syndrome and mitochondrial leukoencephalopathies. In: Magnetic Resonance of Myelination and Myelin Disorders (3rd ed). Berlin Heidelberg: Springer, 2005: 224-244. 